\title{
Site-Specific Chemical Labeling of Long RNA Molecules
}

\author{
Kasper Jahn, ${ }^{\dagger, \ddagger \S}$ Eva Maria Olsen, ${ }^{\dagger, \$, \|}$ Morten Muhlig Nielsen, ${ }^{\ddagger}$, Thomas Tørring, ${ }^{\ddagger, \| l}$ Reza MohammadZadegan,

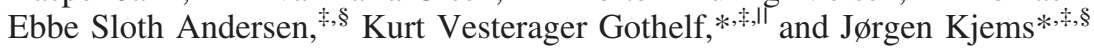 \\ Danish National Research Foundation, Center for DNA Nanotechnology (CDNA) at Interdisciplinary Nanoscience Center \\ (iNANO), Department of Molecular Biology, and Department of Chemistry, Aarhus University, DK-8000 Aarhus, Denmark. \\ Received September 23, 2010; Revised Manuscript Received November 10, 2010
}

\begin{abstract}
Site-specific labeling of RNA molecules is a valuable tool for studying their structure and function. Here, we describe a new site-specific RNA labeling method, which utilizes a DNA-templated chemical reaction to attach a label at a specific internal nucleotide in an RNA molecule. The method is nonenzymatic and based on the formation of a four-way junction, where a donor strand is chemically coupled to an acceptor strand at a specific position via an activated chemical group. A disulfide bond in the linker is subsequently cleaved under mild conditions leaving a thiol group attached to the acceptor-RNA strand. The site-specific thiol-modified target RNA can then be chemically labeled with an optional group, here demonstrated by coupling of a maleimide-functionalized fluorophore. The method is rapid and allows site specific labeling of both in vitro and in vivo synthesized RNA with a broad range of functional groups.
\end{abstract}

\section{INTRODUCTION}

Site-specific labeling of RNA molecules is commonly used for structural and functional studies of RNA $(1,2)$. To this end, the development of solid-phase nucleic acid synthesis has been of great significance; however, limitation in the ability to chemically synthesize long RNAs necessitates methods for direct labeling of natural or longer in vitro synthesized RNA molecules at predetermined internal positions.

To date, a number of methods have been developed to introduce labels site-specifically in long RNA molecules (3). One of the first general strategies reported relies on DNAtemplated ligation of modified, in vitro transcribed, or chemically synthesized RNAs using DNA ligase (4). Similar strategies using RNA ligase have subsequently been reported to increase the yield (5). Another less laborious procedure has been described that involves hybridization of a labeled oligonucleotide probe directly onto the RNA molecules producing a noncovalent label $(6,7)$. Recently, methods have been developed that rely on transfer of a chemical group from a donor oligonucleotide directly onto the target RNA molecule. More specifically, Baum and Silverman developed a site-specific labeling method termed deoxyribozyme-catalyzed labeling (DECAL) in which a DNA enzyme catalyzes the transfer of a $5^{\prime}$-triphosphate end of one RNA molecule onto the 2'-hydroxyl group of a specific nucleoside in the target RNA molecule. This method was successfully applied to demonstrate labeling of a 160-nucleotide large domain of the tetrahymena group I intron (8). The resulting label leaves a branched RNA strand at the target site(s), which can be truncated to 8 bases using the RNA-cleaving DNA

* To whom correspondence should be addressed. Professor Jørgen Kjems, Department of Molecular Biology, Aarhus University, C.F. Mollers Allé, building 1130, DK-8000 Aarhus C, Denmark. Phone: (+45) 8942 2686. Fax: (+45) 8619 6500. E-mail: jk@mb.au.dk. Professor Kurt V. Gothelf, Department of Chemistry, Aarhus University, Langelandsgade 140, DK-8000 C, Denmark. Phone: (+45) 89423907. Fax: (+45)86196199. E-mail: kvg@chem.au.dk.

${ }^{\dagger}$ These authors contributed equally to this work.

* CDNA at iNANO.

$\S$ Department of Molecular Biology.

"Department of Chemistry. enzyme 10-23 (9). Other successful attempts to transfer chemical groups directly from a donor-DNA strand to a short DNA or RNA target strand have been reported $(10,11)$. These methods are based on the transfer of functional groups from 6-thioguanine derivatives to the amine of a Watson-Crick base-paired cytosine residue in the target molecule. This method can potentially also be applied to long RNA molecules.

Although many of the above-mentioned methods have successfully been applied for labeling of RNA, they also have the drawbacks of either being labor-intensive, introducing bulky nucleotide-containing labels, potentially compromising structure and function of the target molecule, or requiring the laborious synthesis of modified nucleoside phosphoramidites. To address these issues, we have devised a novel general method involving a four-way junction complex composed of the target RNA, a universal donor-DNA strand, and two site-specific DNA guide strands. This design results in the chemically synthesized donorDNA strand being independent of the RNA target sequence, eliminating elaborate synthesis for different target sites (12) (Figure 1). Hence, our method provides a highly flexible and cost-competitive strategy for functionalizing long RNA at internal positions, and has the advantage of only attaching a very short linker to the target RNA.

\section{EXPERIMENTAL PROCEDURES}

Materials. All DNA/RNA strands were purchased from DNA Technology A/S, Denmark. Dithiobis(succinimidylpropionate) (DSP) was purchased from Pierce Protein Research Products, USA. The internal amino modifier (Amino modifier C6 dT) was purchased from Glen Research. Alexafluor 568-maleimide was purchased from Molecular Probes. 4-(4,6-Dimethoxy-1,3,5triazin-2-yl)-4-methyl-morpholiniumchloride (DMTMM) was obtained from Acros. Locked nucleic acid (LNA) guide strands were purchased from Ribotask, Denmark.

The strands used for labeling of short RNAs include (unpaired base in the middle of the four-way junction is underlined; superscript "L" denotes LNA base) the following: guide strand, 5'GCGACGTAAATCTTCCAGAGTCGGT; guide strand 2, 5'CCAGATCCGGTGACTGGCCACAAGTG; LNA guide strand 1, 5' GLCGA $^{\mathrm{L} C G T}{ }^{\mathrm{L}} \mathrm{AAA}^{\mathrm{L}}$ TCTTCCAGAGTCGGT; LNA guide 


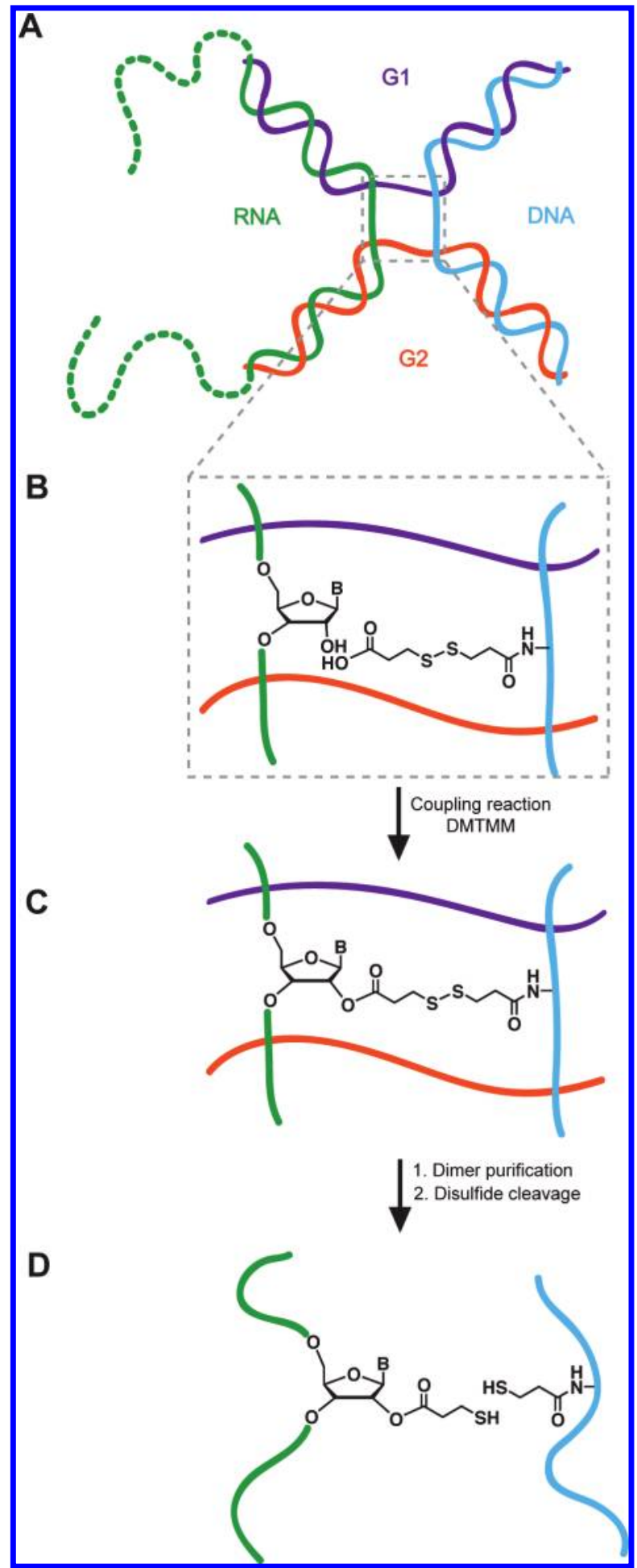

Figure 1. Labeling strategy. (A) Four-way junction containing the acceptor-RNA (green), two guide strands (G1 and G2, purple and orange, respectively) and the donor-DNA (blue). The dashed line indicates potential extensions of the RNA strand. (B) Enlargement below shows the applied chemistry at the center of the four-way junction. (C) Chemical structure of the ester product resulting from the DMTMM coupling to the $2^{\prime} \mathrm{OH}$ of the RNA. (D) Acceptor-RNA and donor-DNA after disulfide cleavage of the purified crosslinking product results in two thiol groups.

strand 2, $5^{\prime}$ CCAGATCCGGTGACTG ${ }^{\mathrm{L}} \mathrm{GCC}^{\mathrm{L}} \mathrm{ACA}^{\mathrm{L}} \mathrm{AGT}^{\mathrm{L}} \mathrm{G}$; short RNA, 5'ACUUGUGGCCBUUUACGUCGC (B: variable target base); short DNA, 5'ACTTGTGGCCBTTTACGTCGC (B: variable target base); universal donor DNA, 5'ACCGACTCTGGAAGtGTCACCGGATCTGG (modified thymine is in lower case).
Strands used for labeling of long HIV-1 RNAs (unpaired base in the middle of the four-way junction is underlined; superscript "L" denotes LNA nucleotides) are as follows: HIV target, 5'ACUUGAAAGCGAAAGGGAAAC; HIV guide strand 1, 5'GTTTCCCTTTTCTTCCAGAGTCGGT; HIV guide strand 2, 5'CCAGATCCGGTGACGGCTTTCAAGT; LNA HIV guide strand $1,5^{\prime} \mathrm{G}^{\mathrm{L}} \mathrm{TTT}^{\mathrm{L}} \mathrm{CCC}^{\mathrm{L}} \mathrm{TTT}^{\mathrm{L}}{ }^{\mathrm{T} C T T C C A}$ GAGTCGGT; LNA HIV guide strand 2, 5' CCAGATCCGGTGACGG ${ }^{\mathrm{L}} \mathrm{CTT}^{\mathrm{L}} \mathrm{TCA}^{\mathrm{L}} \mathrm{AG}^{\mathrm{L}} \mathrm{T}$; universal donor-DNA, 5'ACCGACTCTGGAAGtGTCACCGGATCTGG (modified thymine is in lower case) The HIV-1 leader RNA sequence (HXB2 GenBank: K03455.1) (target area underlined, target base in upper case) is as follows: 5'ggucucucugguuagaccagaucugagccugggagcucucuggcuaacuagggaacccacugcuuaagccucaauaaagcuugccuugagugcuucaaguagugugugeccgucuguugugugacucugguaacuagagaucccucagacccuuuuagucaguguggaaaaucucuagcaguggcgeccgaacagggacuugaaaGcgaaagggaaaccagaggagcucucucgacgcaggacucggcuugcugaagcgcgcacggcaagaggcgaggggcggcgacuggugaguacgccaaaaauuuugacuagcggaggcuagaaggagagagaugggugcgagagcgucaguauuaagcgggggagaauuagaucgaugggaaaaaauucgguuaaggccagggggaaagaaaaaauauaaauuaaacauauaguaugggcaagcagggauccucuagagucgaccugcaggcaugcaagcuug.

Synthesis of Universal Donor-DNA Strand. The donorDNA strand was prepared from an internally modified DNA strand with the DSP linker positioned at the amino-modified base (T) (see Supporting Information Figure S1). A solution containing amino-modified DNA $(130 \mu \mathrm{M}), \mathrm{DSP}(16 \mathrm{mM}), \mathrm{Et}_{3} \mathrm{~N}$ $\left(200 \mathrm{nM}\right.$ ) in 2:2:1 $\mathrm{H}_{2} \mathrm{O} /$ dimethylformamide (DMF)/ $\mathrm{CH}_{3} \mathrm{CN}$ was left to react for $30 \mathrm{~min}$ at room temperature. Water $(100 \mu \mathrm{L})$ was added to the reaction and the aqueous phase washed with dichloromethane $(3 \times 100 \mu \mathrm{L})$. The water phase was lyophilized and the resulting pellet redissolved in $\mathrm{H}_{2} \mathrm{O}(20 \mu \mathrm{L})$ and purified by RP-HPLC (eluent: $\mathrm{A}=$ TEAA $0.1 \mathrm{M}, \mathrm{B}=\mathrm{CH}_{3} \mathrm{CN}, 0-20 \%$ over $20 \mathrm{~min}$, flow $=1 \mathrm{~mL} / \mathrm{min} \mathrm{rt}=16.48 \mathrm{~min}$.). The product was identified by MALDI-TOF MS: [M]; Calcd 9264.9, found 9265. The concentration of the product was determined by UV (NanoDrop) and the overall yield was $25 \%$.

Radioactive Labeling. All radioactive $5^{\prime}$-end labeling reactions were performed in $20 \mu \mathrm{L}$, with $1 \mu \mathrm{L} \mathrm{PNK}$ (polynucleotide kinase) from New England Biolabs, $1 \times$ PNK buffer, ${ }^{\gamma 32} \mathrm{P}$-ATP $(0.5 \mu \mathrm{L}, 6000 \mathrm{Ci} / \mathrm{mmol})$, and 400 pmol DNA or RNA.

Annealing of the Four-Way Junction. The four oligonucleotides were annealed at a 1:1:1:1 ratio in an annealing buffer $(100 \mathrm{mM} \mathrm{NaCl}, 12.5 \mathrm{mM}$ MOPS, $\mathrm{pH}=7.4)$ by heating to 90 ${ }^{\circ} \mathrm{C}$ and slowly cooling to room temperature.

Donor/Acceptor Cross-Linking Product Formation. The coupling reactions were performed on the annealed four-way junction product in a coupling buffer $(12.5 \mathrm{mM}$ MOPS, $\mathrm{pH}=$ $7.4,100 \mathrm{mM} \mathrm{NaCl}$ ) with $50 \mathrm{mM}$ DMTMM as an activator. The coupling reaction was left at room temperature for $2 \mathrm{~h}$. For reactions containing LNA guide strands, the incubation was performed at $40{ }^{\circ} \mathrm{C}$ if not denoted otherwise. For reactions containing DNA guide strands, the reactions were performed at room temperature. The reaction yield was quantified as the amount of RNA in the cross-linked product divided by the total amount of RNA.

Disulfide Cleavage with DTT/TCEP. Donor-DNA/acceptorRNA cross-linking product with a radioactive $5^{\prime}$ label on the RNA strand was purified by denaturing polyacrylamide gel electrophoresis (PAGE), and DTT was added to a final concentration of $5 \mathrm{mM}$ in a Tris- $\mathrm{HCl}$ buffer $(10 \mathrm{mM}, \mathrm{pH}=$ 7.4) or $10 \mathrm{mM}$ tris(2-carboxyethyl)phosphine (TCEP) in a Tris$\mathrm{HCl}$ buffer $(100 \mathrm{mM}, \mathrm{pH}=7.4)$. The released RNA was analyzed on an $8 \%$ denaturing gel PAGE.

Enzymatic Synthesis of HIV-1 Leader RNA. The RNA was prepared from a dsDNA template using Ambion MegaScript T7 


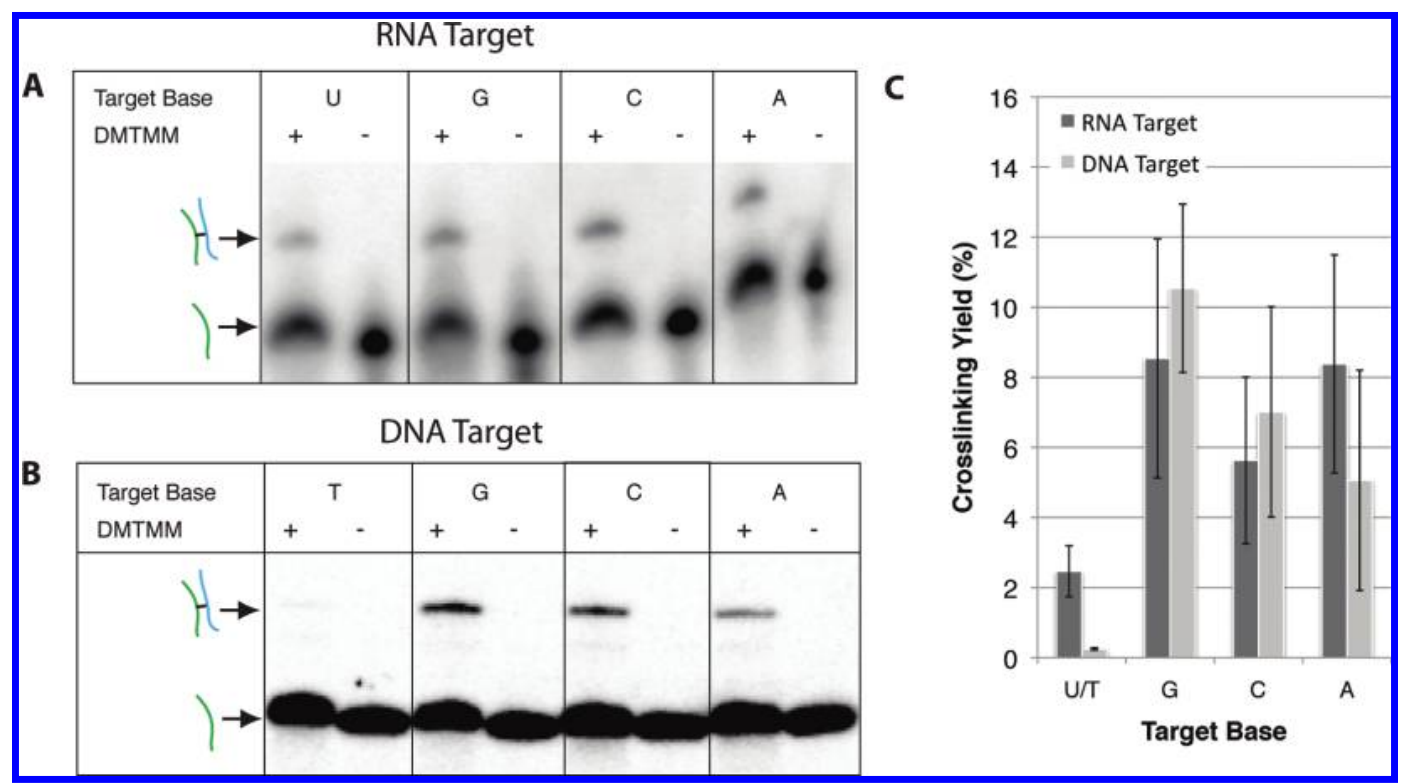

Figure 2. Characterization of target nucleotide in the labeling reaction. (A) Denaturing gel showing that the yield of donor-DNA/acceptor-RNA crosslinking product formation to all four target bases. The acceptor-RNA is radioactively labeled at the $5^{\prime}$ terminal prior to the reaction. (B) Denaturing gel showing the same reaction but targeted to a radioactively $5^{\prime}$-end labeled DNA. (C) Quatification of the crosslinking yield and the dependence on target base using short target strands.

kit according to the standard protocol. After DNaseI treatment, the RNA was gel-purified on a 5\% denaturing PAGE gel.

Primer Extension. PAGE-purified site-specifically modified HIV-1 leader RNA (0.5 pmol) and equimolar amounts of a ${ }^{32} \mathrm{P}$ 5'-labeled primer (5'-CCGAGTCCTGCGTCGAGA) were annealed in $6 \mu \mathrm{L}$ annealing buffer (10 mM Tris, $\mathrm{pH}=7.4,40$ $\mathrm{mM} \mathrm{KCl}$ ). The radioactive primer binds approximately 30 base pairs away from the expected modification site. The mixture was incubated at $90{ }^{\circ} \mathrm{C}$ for $3 \mathrm{~min}$ followed by $50{ }^{\circ} \mathrm{C}$ for 25 min. Reverse transcriptase reagents ( 1 unit of AMV Reverse Transcripase from Fermentas, $1 \times$ running buffer, NTPs $(200$ $\mu \mathrm{M}$ of each)) were added to a final volume of $10 \mu \mathrm{L}$. The extension reaction was incubated at $37^{\circ} \mathrm{C}$ for $30 \mathrm{~min}$, stopped, and precipitated by adding $\mathrm{NaOAc}(40 \mu \mathrm{L}, 0.3 \mathrm{M})$ and $21 / 2$ vol ethanol (96\%).

Sequencing Reactions. HIV-1 leader RNA (5 pmol) was incubated with ${ }^{32} \mathrm{P}$ end-labeled primer (5'-CCGAGTCCTGCGTCGAGA; $2.5 \mathrm{pmol})$ in an annealing buffer $(100 \mathrm{mM}$ $\mathrm{NaCl}, 10 \mathrm{mM}$ Tris- $\mathrm{HCl}, \mathrm{pH}=7.4,2.5 \mathrm{mM}$ EDTA) in a volume of $21 \mu \mathrm{L}$. The mixture was heated to $90{ }^{\circ} \mathrm{C}$ for $3 \mathrm{~min}$ and incubated at $50{ }^{\circ} \mathrm{C}$ for $25 \mathrm{~min}$. The mixture was divided into 4 tubes with $5 \mu \mathrm{L}$ in each tube. Primer extensions were performed in a total volume of $10 \mu \mathrm{L}$ by adding sequencing mixture to final concentrations of 1 unit of AMV RT (Fermantas), $1 \times$ Running Buffer, NTPs (200 nM of each), and different ddNTP (Amersham Biosciences) set to the following concentrations: ddATP, 200 nM; ddTTP, 200 nM; ddCTP, 66 nM; ddGTP, 133 $\mathrm{nM}$. The reaction was incubated at $37{ }^{\circ} \mathrm{C}$ for $30 \mathrm{~min}$ and precipitated by adding NaOAc $(10 \mu \mathrm{L}, 0.5 \mathrm{M})$ and ethanol $(50$ $\mu \mathrm{L}, 96 \%)$. The samples were redissolved in a Maxam-Gilbert loading buffer (90\% formamide, $1 \mathrm{mM}$ EDTA, $10 \mathrm{mM} \mathrm{NaOH}$ ).

Fluorophore Coupling. The gel-purified HIV-1 leader RNA/ donor-DNA cross-linking product (1 pmol) was cleaved with TCEP $(8 \mu \mathrm{L}, 10 \mathrm{mM})$ in a Tris-HCl buffer $(100 \mathrm{mM}, \mathrm{pH}=7.4)$ for 45 min. Thereafter, a solution of Alexafluor 568-maleimide $(2 \mu \mathrm{L}, 1$ $\mathrm{mM}$ ) was added to the solution. The reaction was incubated at room temperature for $2 \mathrm{~h}$ and purified on a G-50 Sephadex column to remove excess of free fluorophore. The product was separated on a $7 \%$ denaturing PAGE gel in $1 \times$ TBE buffer, and scanned on a Typhoon scanner (GE Healthcare) to quantitate the incorporation of fluorescence. Settings on the Typhoon scanner were as follows: sensitivity $=$ normal, laser $=532 \mathrm{~nm}$, filter $=610 \mathrm{~nm} / 30$, photomultiplier $=550$.

\section{RESULTS}

Design of the Reaction Complex. A tetrameric DNA-RNA complex was designed to enable the transfer of a chemical group from a labeled DNA strand onto a nonlabeled RNA molecule in a site-specific manner. The method relies on the 4-(4,6dimethoxy-1,3,5-triazin-2-yl)-4-methyl-morpholinium chloride (DMTMM)-catalyzed formation a bond between the carboxylic acid-containing DNA-donor molecule and the 2 '-OH of a ribose in the target RNA by activation of the carboxylic acid. Carboxylic acids are efficiently activated by DMTMM to form esters with primary and secondary alcohols (13). The positioning of the carboxylic acid and the nonpaired target nucleotide in close proximity in the four-way junction should favor the reaction (12).

The donor molecule is formed by reacting an amino-modified DNA strand with the commercially available DSP-linker containing a cleavable disulfide spacer with an $\mathrm{N}$-hydroxysuccinimide (NHS)-ester at each end. The reaction results in the formation of an amide linkage between the amino group at the DNA and one of the reactive NHS esters. Hydrolysis of the other NHS-ester results in a carboxylic acid at the end of the linker (Figure 1B and Supporting Information Figure S1). During the donor strand synthesis, no dimer product was formed due to the large excess of the DSP linker. The donor-DNA strand is easily purified by reverse-phase HPLC. Importantly, the fourway junction structure promotes close proximity of the acidcontaining linker to the target site on the RNA without the need for direct base pairing between the modified DNA and target RNA. Two unmodified DNA guide strands that are complementary to the sequences flanking the intended labeling site in the RNA at one end and to the sequences flanking the chemical modification of the DNA donor at the other end were designed to facilitate assembly of the complex. (Figure 1A).

Site-Specific Labeling of Short RNA. To investigate the feasibility and specificity of the method, we first applied it to a short radiolabeled RNA molecule of 21 nucleotides using guide DNAs complementary to positions $1-10$ and $12-21$. Characterization of the reaction products on a denaturing gel revealed 


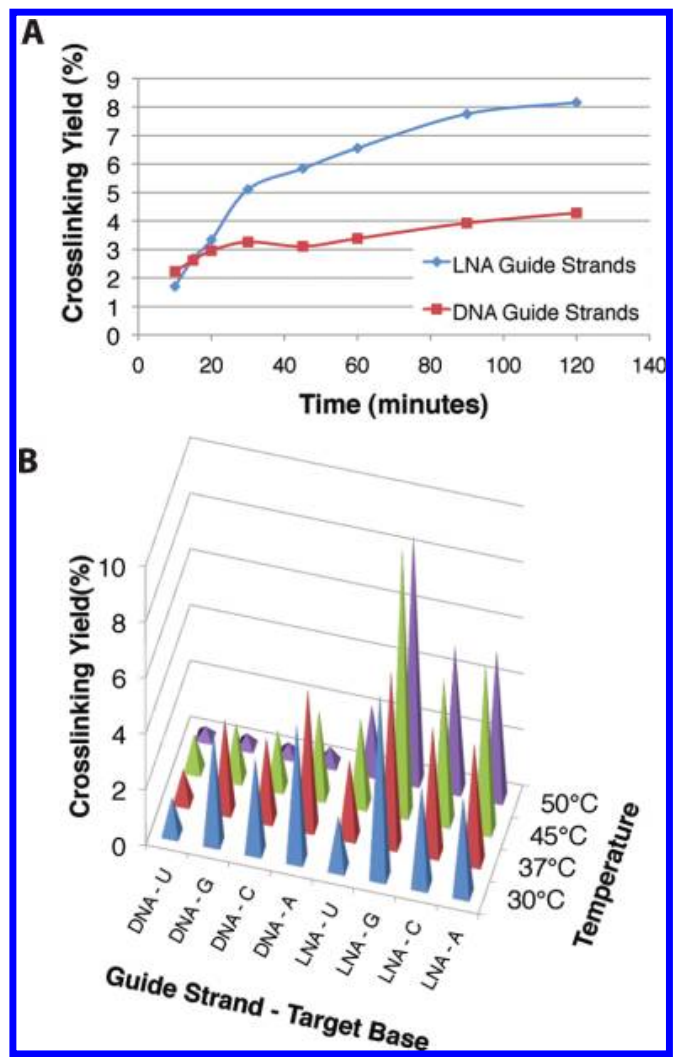

Figure 3. Optimization of product yields (A) Graph showing the crosslinking efficiency between donor-DNA and acceptor-RNA when using LNA guide strands and DNA guide strands as a function of time. The yield is quantified as the amount of RNA in the crosslinking product divided by the total amount of RNA. (B) Crosslinking efficiency between donor-DNA and acceptor-RNA as a function of temperature and target base identity.

a slowly migrating band dependent on the presence of DMTMM (Figure 2A). To investigate the influence of the targeted nucleotide identity, RNA oligos with 4 different bases at the target site were prepared. The appearance of cross-linked product in all reactions of similar intensity indicates that the method is compatible with all 4 ribonucleotides at the target site (Figure 2A).

Using DNA guide strands, the yield of the DMTMM activated esterification was approximately $4 \%$. To improve the yield of the cross-linking reaction, we introduced 4 LNA residues in each of the DNA guide strands. LNA-RNA duplexes have a higher melting temperature than DNA-RNA duplexes (14), and the use of LNA could potentially improve the yield by increasing the complex stability and furthermore competing with internal secondary structure in the RNA. As predicted, a significant increase in the yield of the cross-linked product to approximately $8 \%$ can be seen after $2 \mathrm{~h}$ (Figure $3 \mathrm{~A}$ ). The cross-linking reaction, using LNA-modified guide strands, was studied more extensively with respect to temperature, target base identity, and LNA modification in guide strands (Figure $3 \mathrm{~B}$ ). From these data, we conclude that the use of LNA guide strands generally increases the yield over a wide range of temperatures, and that a maximum is reached when the reaction temperature is raised to $45-50$ ${ }^{\circ} \mathrm{C}$. In contrast, the yield decreases at higher temperature when using nonmodified DNA guide strands, most likely as a result of denaturation of the four-way junction at elevated temperature.

To study the specificity of the reaction of the activated carboxylic acid with the $2^{\prime}$ hydroxyl of the RNA ribose, we investigated the cross-linking efficiency with all 4 DNA residues that lack the $2^{\prime}$-hydroxyl. It is well-known that DMTMMactivated carboxylic acids also can react very efficiently with aliphatic amines to form amides (13). We therefore investigated whether it also would react with the less reactive 2-aminopyridine-type amino groups on adenine, guanine, and cytosine. Figure 2B,C shows that the DMTMM-activated carboxylic acid reacts efficiently with guanine, cytosine, and adenosine, but not with thymine, which is expected based on the lack of both the 2 ' hydroxyl and amine groups. This indicates that the carboxylic acid can react with a specific 2-aminopyridine-type amine in the target base. The carboxylic acid can also react with the $2^{\prime}$ $\mathrm{OH}$ on the RNA, based on the reactivity of uracil targets, which lack an amine.

Importantly, the absence of any reaction product when thymine is present at the target site clearly indicates that no other position in the nucleotide is reactive. Furthermore, it also shows that the unpaired target nucleotide is the sole reactive site, since no cross-linking product was observed with the adjacent cytosine or other residues (Figure 2B,C). This clearly demonstrates the nucleotide specificity of the technique.

The ratio between the donor-DNA and acceptor-RNA was also varied, to see if this affected the yield of the reaction (Supporting Information Figure S2). Increasing the amount of donor-DNA of up to 10-fold excess increases the yield only 1.3-fold. However, since the excess of donor-DNA may potentially give rise to side reactions to other nucleotides in the RNA by binding to homologous sites, we recommend not to apply the donor-DNA in excess.

To verify that the more slowly migrating band corresponds to the cross-linked RNA-DNA species, the band was extracted from the gel and treated with the reducing agent DTT (15) or TCEP (16) to cleave the disulfide in the DSP linker, followed by analysis on a denaturing gel. Under both conditions, the more slowly migrating band disappeared, confirming the presence of the disulfide link provided by the donor DNA (Supporting Information Figures S3 and S4, respectively). DNase I treatment also resulted in a band migrating similarly to the RNA target strand alone, implying that RNA was exclusively cross-linked to DNA (Supporting Information Figure S5). Furthermore, the reaction was dependent on the presence of both guide strands demonstrating the importance of the four-way junction for product formation (data not shown).

Specific Labeling of the HIV-1 Leader RNA. To test the labeling method to a biological relevant RNA, we applied it to a long, naturally occurring RNA sequence, encompassing 488 nucleotides at the $5^{\prime}$ end of the HIV-1 RNA genome. The guide strands were designed to target the internal position 206 shown to be accessible to hybridization in previous studies (17), as well as with a SHAPE (selective 2'-hydroxyl acylation analyzed by primer extension) analysis of the HIV genomic RNA (18). As can be seen in Figure 4A, DNA-RNA cross-linked product is formed with an efficiency of approximately $1 \%$ when DNA guide strands are used. The use of LNA guide strands increased the yield of the cross-linking product formation to $4.2 \% \pm 1.2 \%$, which is lower than observed for the short RNA strands (Figure 4B). This could be attributed to the more rigid secondary structure in the HIV-1 RNA that might restrict the access to the labeling site, especially if LNAs are absent from the guides. To further analyze the specificity of the labeling, we performed a primer extension experiment using the gel-purified crosslinking product as template (Figure 4C). A major product corresponding to the transcriptional termination at the position immediately prior to the targeted nucleotide appears in the gel. The minor band observed in the reverse transcription reaction, just below the major product, is most likely a -2 premature stop that is commonly seen in addition to the major -1 stop when chemical modifications are present (19). The labeled site corresponds exactly to the predicted target site demonstrating 


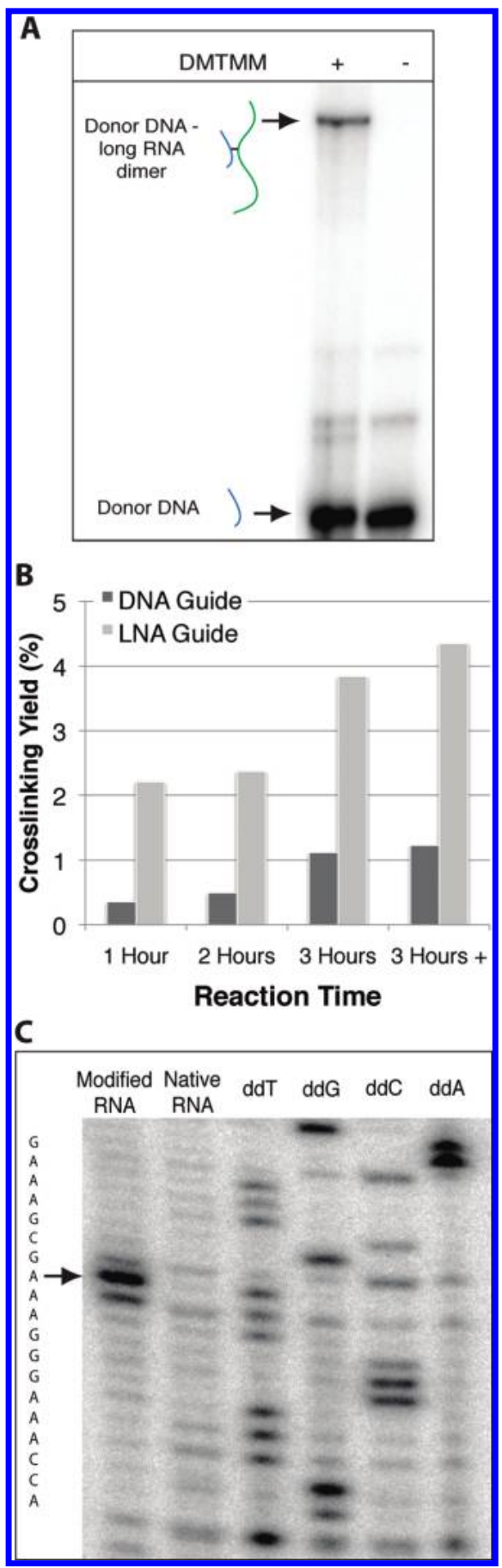

Figure 4. Application of the labeling method to HIV-1 derived RNA (A) Denaturing gel showing the crosslinking product formation between radioactively $5^{\prime}$ end-labeled donor-DNA and a $\sim 500$ bp long HIV-1derived acceptor-RNA. (B) Crosslinking yield depending on time and LNA modification. + indicates that extra DMTMM was added after 2 hours increasing the yield slightly. (C) Autoradiogram showing the primer extension reaction on the crosslinked HIV-1 template. A sequencing ladder is included to indicate that the primer extension reaction terminates almost exclusively at the base prior to the target base.

that the method can be used to label long and highly structured RNA molecules at specific sites.

The free thiol group positioned at the target nucleotide after reduction provides an anchoring point for a number of functional groups. To demonstrate the labeling principle, we selected a maleimide-functionalized fluorophore that reacts efficiently and selectively with thiols (20). Gel-purified product containing the HIV-1 leader RNA cross-linked to the modified donor-DNA strand was first reduced with TCEP (16). TCEP was used to

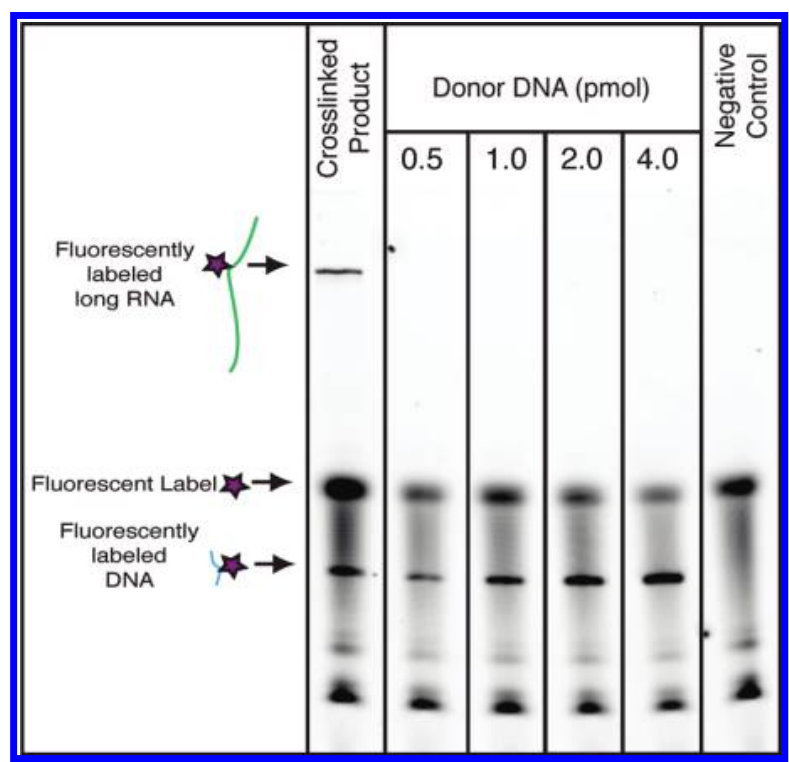

Figure 5. Fluorescent scan of a denaturing gel, showing labeled product obtained using HIV-1 leader RNA as substrate. The fluorophore-coupled HIV-1 RNA, fluorophore-maleimide and donor DNA are indicated to the left of the gel image. Lane 1, conjugation of the maleimidefluorophore to the purified HIV-RNA/Donor-DNA. Lanes 2-5, increasing amounts of donor-DNA reacted with the maleimide-fluorophore. Lane 6, negative control with only the maleimide-fluorophore present.

avoid coupling to the free thiol groups in the DTT that may remain in the reaction. The maleimide-functionalized fluorophore was then coupled to the free thiols on the RNA and donor DNA. As previously noted, cleavage of the cross-linking product with TCEP is a highly efficient reaction and is essentially quantitative. We also tested the efficiency of fluorophore attachment and the yield of the reduction and fluorophore attachment steps combined was determined to be 33\% (Supporting Information Figure S6).

To determine the overall efficiency of the labeling reaction, 40 pmol of HIV-1 RNA and 40 pmol of donor DNA were used as starting materials. The cross-linked RNA-DNA product was gel purified and subsequently reduced with TCEP and conjugated to the fluorophore-maleimide. The product was quantified by comparing it to varying amounts of the donor-DNA strand reacted with the fluorophore-maleimide (Figure 5). A fluorescent scan was performed and the lane profiles were investigated (Supporting Information Figure S7). First, the result verified that the amounts of labeled HIV RNA and short DNA were the same, as would be expected from their 1:1 ratio. Second, when comparing the intensities of the bands in the reaction lane with the control lanes, it is estimated that $1 \mathrm{pmol}$ material was formed, giving an overall yield of the labeling reaction of $2.5 \%$.

\section{DISCUSSION}

The RNA labeling method proposed here solves many of the drawbacks of previously published methods by ultimately attaching a free thiol with a structurally very small linker to a specific nucleotide within a long RNA strand. Importantly, it is possible to label natural RNA extracted from cells, which is not feasible with the templated ligation method. The modified donor DNA strand is easily available in just one conjugation step of amine-modified DNA with the commercially available DSP linker. Furthermore, our method only requires de novo synthesis of two new DNA or LNA guide strands for each new target after establishment of the common donor-DNA. Purification of the labeled product is conveniently done by gel electrophoresis of the cross-linked RNA-DNA sequences prior to chemical reduction of the disulfide bond. 
Despite careful optimization of the method, the yield remained below $10 \%$ for any target investigated. This precludes the method being used as a practical tool for double labeling of RNA. However, both in vitro synthesized RNA and cellular derived RNA are usually available in reasonably large amounts enabling up-scaling of the labeling reaction.

Another important parameter is the specificity of the reaction. Our original intention for the reaction was that it should be specific for the $2^{\prime}-\mathrm{OH}$ position. However, as observed, the donor-DNA also reacts with the 2-aminopyridine-type amine on the target nucleotide, and the end product is a distribution of the two. It is possible to circumvent this if a uridine is chosen as the target nucleotide, as it contains no exocyclic amines. The reaction with the bases, however, does enable labeling of long DNA single strands, which can be useful in the growing field of DNA nanotechnology.

The single band observed in the primer extension assay and the lack of cross-linking product formation when thymine is positioned at the target site suggests that the cross-linking occurs exclusively to the intended nucleotide (Figure 4C). A potential problem is that the labeling site may not be accessible for annealing to the DNA guides, in particular, when targeting highly structured RNA. Using LNA in the guide strands to increase the binding affinity to the target may, to some degree, circumvent this problem. Improved accessibility upon LNA modification has previously been observed using antisense and DNA enzymes in the context of HIV-1 (21).

Another advantage of our method is the incorporation of a free thiol that enables efficient conjugation of functional groups. Here, we demonstrate the attachment of a fluorophore for optical detection, but this method can easily be extended to any other materials that are linked to a thiol-specific cross-linking functionality such as maleimide or vinylsulfones (22). It may also be used for attachment of biotin, reactive cross-linkers for structural probing, larger structures like lipids, peptides, nucleic acids, or even nanoparticles that could provide RNA molecules with new functionalities.

\section{ACKNOWLEDGMENT}

This work was funded by the Danish National Research Foundation, the iNANO Graduate School, and the OChem Graduate School.

Supporting Information Available: Figures S1-S7 as described in the text. This material is available free of charge via the Internet at http://pubs.acs.org.

\section{LITERATURE CITED}

(1) Bokinsky, G., Rueda, D., Misra, V. K., Rhodes, M. M., Gordus, A., Babcock, H. P., Walter, N. G., and Zhuang, X. (2003) Singlemolecule transition-state analysis of RNA folding. Proc. Natl. Acad. Sci. U.S.A 100, 9302-7.

(2) Penedo, J. C., Wilson, T. J., Jayasena, S. D., Khvorova, A., and Lilley, D. M. (2004) Folding of the natural hammerhead ribozyme is enhanced by interaction of auxiliary elements. $\underline{R N A}$ 10, 880-8.

(3) Qin, P. Z., and Pyle, A. M. (1999) Site-specific labeling of RNA with fluorophores and other structural probes. Methods in Enzvmology 18, 60-70.

(4) Moore, M. J., and Sharp, P. A. (1992) Site-specific modification of pre-mRNA: the 2 -hydroxyl groups at the splice sites. Science 256, 992-7.
(5) Stark, M. R., Pleiss, J. A., Deras, M., Scaringe, S. A., and Rader, S. D. (2006) An RNA ligase-mediated method for the efficient creation of large, synthetic RNAs. RNA 12, 2014-9.

(6) Dorywalska, M., Blanchard, S. C., Gonzalez, R. L., Kim, H. D., Chu, S., and Puglisi, J. D. (2005) Site-specific labeling of the ribosome for single-molecule spectroscopy. Nucleic Acids Res. $33,182-9$.

(7) Smith, G. J., Sosnick, T. R., Scherer, N. F., and Pan, T. (2005) Efficient fluorescence labeling of a large RNA through oligonucleotide hybridization. RNA 11, 234-9.

(8) Baum, D. A., and Silverman, S. K. (2007) Deoxyribozymecatalyzed labeling of RNA. Angew. Chem. Int. Ed. Engl. 46, 3502-3504.

(9) Santoro, S. W., and Joyce, G. F. (1997) A general purpose RNA-cleaving DNA enzyme. Proc. Natl. Acad.Sci. U.S.A. 94, 4262-6.

(10) Ali, M., Alam, R., Kawasaki, T., Nakayama, S., Nagatsugi, F., and Sasaki, S. (2004) Sequence- and base-specific delivery of nitric oxide to cytidine and 5-methylcytidine leading to efficient deamination. J. Am. Chem. Soc. 125, 8864-8865.

(11) Onizuka, K., Taniguchi, Y., and Sasaki, S. (2009) Site-specific covalent modification of RNA guided by functionality-transfer oligodeoxynucleotides. Bioconiugate Chem. 20, 799-803.

(12) Hansen, M. H., Blakskjær, P., Petersen, L. K., Hansen, T. H., Højfeldt, J. W., Gothelf, K. V., and Hansen, N. J. (2009) A yoctoliter-scale DNA reactor for small-molecule evolution. J. Am. Chem. Soc. 131, 1322-7.

(13) Kunishima, M., Kawachi, C., Morita, J., Terao, K., Iwasaki, F., and Tani, S. (1999) 4-(4,6-Dimethoxy-1,3,5-triazin-2-yl)-4methyl-morpholinium Chloride: an efficient condensing agent leading to the formation of amides and esters. Tetrahedron 55, 13159-13170.

(14) Vester, B., and Wengel, J. (2004) LNA (locked nucleic acid): high-affinity targeting of complementary RNA and DNA. Biochemistry 43, 13233-41.

(15) Cleland, W. W. (1964) Dithiothreitol, a new protective agent for SH groups. Biochemistry 3, 480-482.

(16) Burns, J. A., Butler, J. C., Moran, J., and Whitesides, G. M. (1991) Selective reduction of disulfides by tris(2-carboxyethyl)phosphine. J. Org. Chem. 56, 2648-2650.

(17) Damgaard, C. K., Andersen, E. S., Knudsen, B., Gorodkin, J., and Kjems, J. (2004) RNA interactions in the $5^{\prime}$ region of the HIV-1 genome. J. Mol. Biol. 336, 369-379.

(18) Wilkinson, K. A., Gorelick, R. J., Vasa, S. M., Guex, N., Rein, A., Mathews, D. H., Giddings, M. C., and Weeks, K. M. (2008) High-throughput SHAPE analysis reveals structures in HIV-1 genomic RNA strongly conserved across distinct biological states. PLoS Biol. 6, e96.

(19) Kjems, J., Brown, M., Chang, D. D., and Sharp, P. A. (1991) Structural analysis of the interaction between the human immunodeficiency virus Rev protein and the Rev response element. Proc. Natl. Acad. Sci. U.S.A. 88, 683-7.

(20) Ghosh, S. S., Kao, P. M., McCue, A. W., and Chapelle, H. L. (1990) Use of maleimide-thiol coupling chemistry for efficient syntheses of oligonucleotide-enzyme conjugate hybridization probes. Bioconiugate Chem. 1, 71-76.

(21) Jakobsen, M. R., Haasnoot, J., Wengel, J., Berkhout, B., and Kjems, J. (2007) Efficient inhibition of HIV-1 expression by LNA modified antisense oligonucleotides and DNAzymes targeted to functionally selected binding sites. Retrovirology 4, 29.

(22) Højfeldt, J. W., Blakskjær, P., and Gothelf, K. V. (2006) A cleavable amino-thiol linker for reversible linking of amines to DNA. J. Org. Chem. 71, 9556-9.

BC100422K 